CASE REPORT

\title{
"Surgery-first Orthognathic Approach" for Correction of Skeletal Class III with Open Bite
}

\author{
Reshme Radha Divakar ${ }^{1}$, Ratna Parameswaran², Janani Jayapal ${ }^{3}$, Devaki Vijayalakshmi ${ }^{4}$
}

\begin{abstract}
Introduction: This case report illustrates the treatment of a skeletal class III patient with the surgery-first orthognathic approach (SFOA) protocol. Background: "Surgery-first orthognathic approach" is a growing trend that provides an immediate facial change. The SFOA protocol utilizes dentoalveolar compensation as the key advantage to effect immediate facial change, which in conjunction with the rapid acceleratory phenomenon (RAP) augments the intended tooth movement, thereby reducing the total treatment duration.

Case description: A 24-year-old male presented with forwardly placed lower jaw with associated difficulty during eating. Clinical examination revealed severe lip incompetency, mandibular excess, class III skeletal malocclusion, severe bimaxillary incisor proclination, posterior crossbite, and lower midline shift toward right on an average mandibular plane angle.

Conclusion: The SFOA protocol involving bilateral sagittal split osteotomy (BSSO) mandibular setback along with the correction of the pitch and yaw was performed and the orthodontic phase was followed.

Clinical significance: The total treatment time was 5 months and 15 days, following which excellent facial transformation and stable occlusion was achieved.
\end{abstract}

Keywords: Class III skeletal base, Orthognathic surgery, Rapid acceleratory phenomenon, Surgery-first orthognathic approach. Journal of Contemporary Dentistry (2019): 10.5005/jp-journals-10031-1266

\section{BACKGROUND}

Conventional orthognathic surgery treatment always involves an exhaustive period of presurgical treatment and postsurgical orthodontic treatment, which amounts to a total treatment period of 3-4 years. ${ }^{1}$ The surgery-first orthognathic approach (SFOA) implies orthognathic surgery being carried out first, followed by orthodontic treatment to align the teeth and settle the occlusion. The SFOA is a growing trend toward implementing a treatment plan that provides instantaneous facial change and has revolutionized the orthognathic approach. ${ }^{2,3}$ In surgery-first treatment protocols, the primary objective involves surgical correction of jaws using the dentoalveolar compensations to our advantage, thereby the presurgical orthodontic treatment phase is eliminated or reduced..$^{4-6}$ In 1991, Brachovagel proposed this method as the most desirable orthognathic approach as it promotes rapid upgrade in facial esthetics, thus enhancing psychosocial benefits and dramatically reducing the treatment time. ${ }^{7}$

Following orthognathic surgery, a period of rapid metabolic activity within tissues ensues, known as the regional acceleratory phenomenon (RAP). By performing surgery first, RAP can be utilized to facilitate efficient orthodontic treatment. This phenomenon is believed to be a key factor in the notable reduction in treatment duration using SFOA. The postsurgical orthodontic phase therefore requires minimal duration for completion..$^{8,9}$ This case report illustrates the treatment of a skeletal class III patient using SFOA with the benefit of the regional accelerated phenomenon.

\section{Case Description}

A 24-year-old patient had reported to the Department of Orthodontics and Dentofacial Orthopedics with the chief complaint of forwardly placed lower jaw and difficulty in chewing food.
${ }^{1-4}$ Department of Orthodontics and Dentofacial Orthopedics, Meenakshi Ammal Dental College and Hospital, Chennai, Tamil Nadu, India

Corresponding Author: Ratna Parameswaran, Department of Orthodontics and Dentofacial Orthopedics, Meenakshi Ammal Dental College and Hospital, Chennai, Tamil Nadu, India, Phone: +91 9176312366, e-mail: rathu07@yahoo.co.in

How to cite this article: Divakar RR, Parameswaran R, Jayapal J, et al. "Surgery-first Orthognathic Approach" for Correction of Skeletal Class III with Open Bite. J Contemp Dent 2019;9(3):144-149.

Source of support: Nil

Conflict of interest: None

Extraoral examination revealed an anterior divergent facial pattern, concave facial profile, an acute nasolabial angle, and an average mandibular plane angle. Intraoral examination revealed a bilateral posterior cross-bite accompanied by a reverse overjet of $4 \mathrm{~mm}$ along with midline shift of $2 \mathrm{~mm}$ toward the right side. A curve of Spee of $2 \mathrm{~mm}$ and $1 \mathrm{~mm}$ was recorded on the right and left side, respectively. A class III molar and canine relationship was evident on the right and left side (Fig. 1).

Routine investigative procedures revealed a missing 28 and no other significant pathology. Model analysis pointed out to the case being on the borderline of the extraction treatment protocol. Cephalometric measurements highlight the proclination in the maxillary and mandibular segment in consort with a significant negative correlation of the skeletal arrangement (ANB $-6^{\circ}$ ). Horizontal discrepancies were evident in the mandibular segment with an increase in the length [Go-Me $83 \mathrm{~mm}$ ] [Go-Pog $87 \mathrm{~mm}$ ]. However, the maxillary segment measurements were within the norm. 

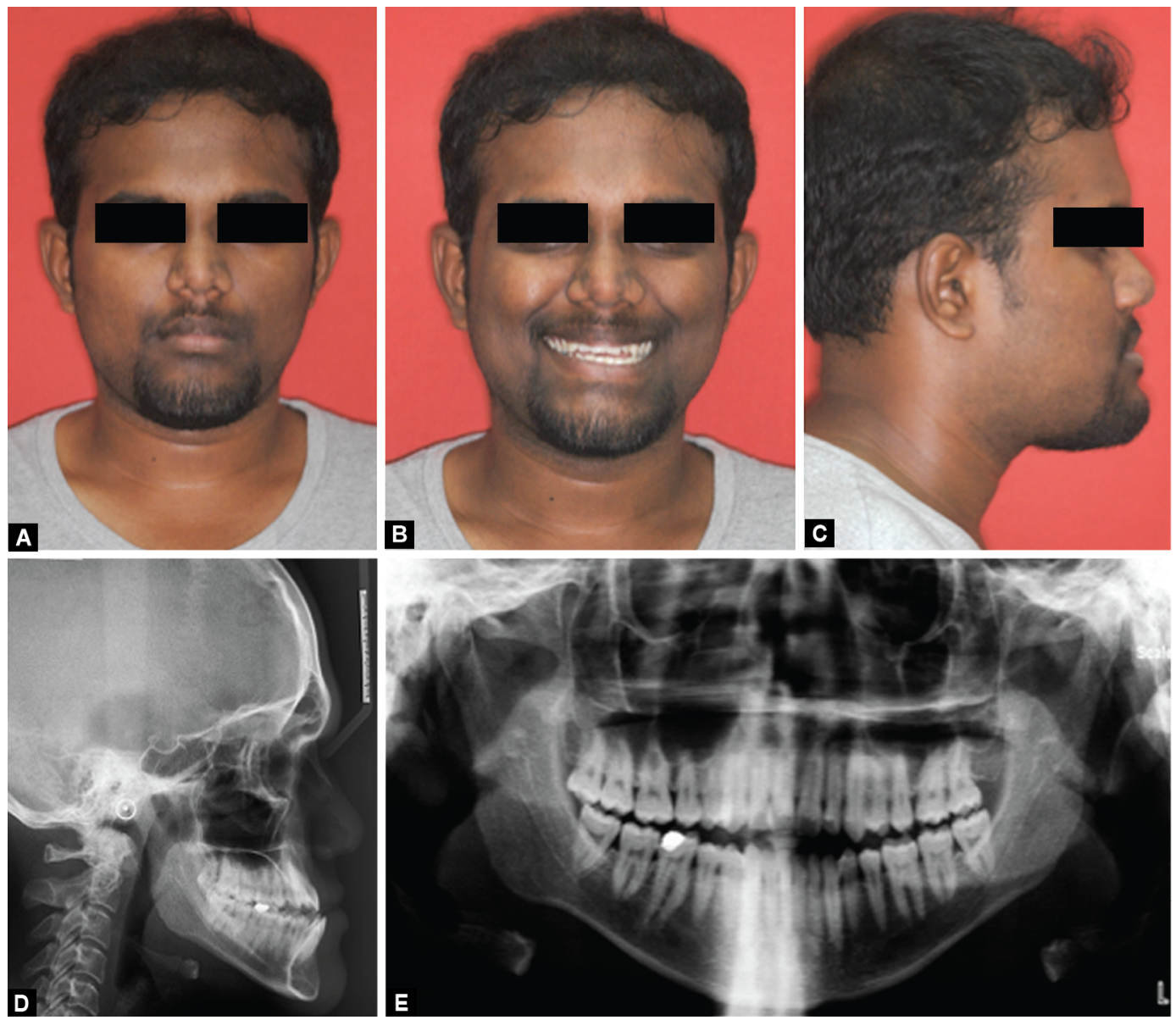

Figs $1 \mathrm{~A}$ to $\mathrm{E}$ : Pretreatment extraoral photographs and radiographs

Contingent with the clinical inspection and the investigative data, the patient was diagnosed as Angle's class III dentoalveolar malocclusion on class III skeletal base accredited to the orthognathic maxilla and prognathic mandible with a posterior crossbite, reverse overbite, and negative overjet accompanied with an open bite in the anterior segment on an average mandibular plane angle.

\section{Aims and Objectives}

- To improve facial esthetics

- To achieve ideal soft tissue profile

- To correct the skeletal discrepancy

- To align the upper and lower arch

- To achieve ideal overbite and overjet

- To achieve class I molar and class I canine relation

- To correct the bilateral posterior scissors bite

- To correct the midline discrepancy

\section{Treatment Plan}

Evaluating the severity of the skeletal malocclusion the patient was presented with two treatment options, the first being the SFOA protocol that involves mandibular setback by bilateral sagittal split osteotomy (BSSO) of $8 \mathrm{~mm}$ combined with an anterior pitch up of $2 \mathrm{~mm}$ followed by orthodontic therapy with an overall predicted treatment duration of 6 months.
The second option was the conventional orthodontic-surgical therapy involving a pretreatment orthodontic phase followed by surgical correction of skeletal bases and postsurgical orthodontic phase for settling and refinement of occlusion. The overall suggested treatment duration was 20 months. The patient chose the SFOA treatment option owing to better facial improvements in the early stages of treatment and reduced treatment duration.

\section{Treatment Progress}

In the current case, the treatment was commenced with the bonding of orthodontic brackets $(0.022 \times 0.028 \mathrm{MBT}$ prescription) along with a passive ligation of the 0.012 SS engaged in the upper and lower arches. A surgical treatment objective (STO) was simulated using Facad $^{\oplus}$ to determine the extent of correction necessary to alleviate the skeletal discrepancy. The surgical splint was fabricated after the face bow transfer and articulation using SAM III articulator once the mock surgery was done as per the STO. The intended transitional malocclusion (ITM) was established in the surgical splint for the three-point contact necessary for a stable occlusal table post the surgical phase. The ITM so obtained following mock surgery developed an open bite in the posterior region.

A BSSO with a setback of the mandibular segment by $8 \mathrm{~mm}$ and an anterior pitch up of the mandible by $2 \mathrm{~mm}$ was done to alleviate the open bite and the segments were plated with the surgical splint in place. 
Once the class I skeletal base was achieved surgically, the alignment and levelling phase of treatment was commenced with $0.016 \mathrm{NiTi}$ wire a week after to exploit the RAP (Fig. 2). The posterior crossbite evident in relation to 27,37 was corrected using intermaxillary elastics. Dentoalveolar extrusion was achieved using the fabricated segmental extrusion arch in relation to 11, 12, 21, 22 using a $16 \times 22$ SS wire (Fig. 2). Following this, a regular sequence of wire upgradation was done for occlusal detailing. After the total treatment time of 5 months and 15 days, the orthodontic phase was completed. Upper and lower Essix was placed in order to maintain the intruded molars in the same vertical relation.

\section{Discussion}

In 1988, Behrman introduced the concept of "surgery first and orthodontics second," which was also named as surgery-first approach (SFA). They hypothesized that once the jaw position was counterbalanced, the normalized soft tissues would facilitate the postoperative tooth movement and thus shorten the duration of treatment. Based on this approach a preplanned orthodontic treatment is fulfilled after the surgery transforming facial esthetics, thereby swiftly diminishing the total treatment time. ${ }^{10}$

The SFA was developed chiefly to improve patient care by reducing treatment duration. Hence, the first indication for any SFA should be patient demand. The indications for the surgeryfirst patient include (1) minimal crowding in the anterior teeth, which would not require extensive preoperative orthodontics; (2) favorable curve of Spee-patients with flat-to-mild curve of Spee will be considered for the SFA; (3) normal range of angle between the basal bone to upper and lower incisors; (4) minimal transverse discrepancies; (5) pronounced soft tissue imbalance in skeletal class III patients; (6) cases in which decompensation is not required; (7) patients who want immediate esthetic result or who want to improve both function and esthetics; (8) at least three stable occlusal stops with positive overbite of six anterior teeth
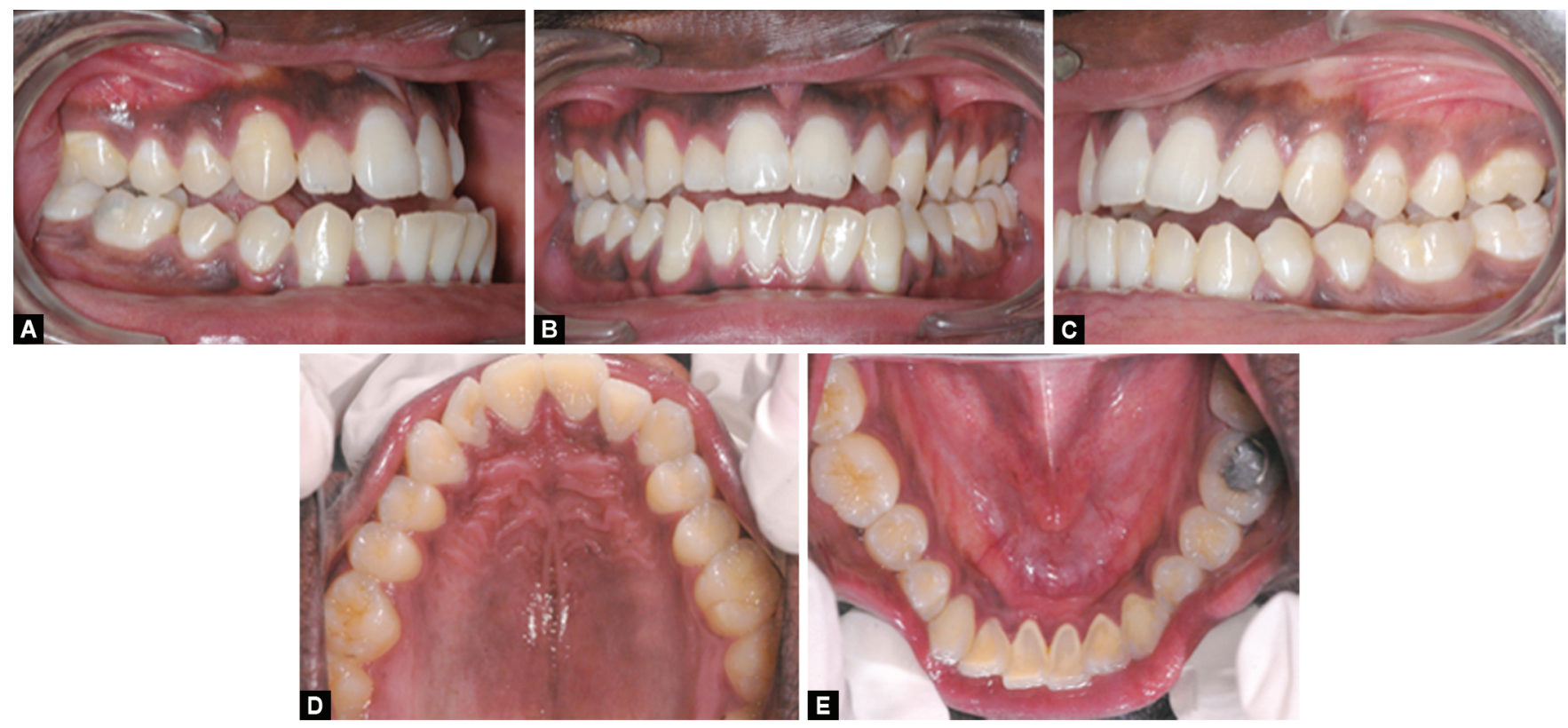

Figs 2A to E: Pretreatment intraoral photographs
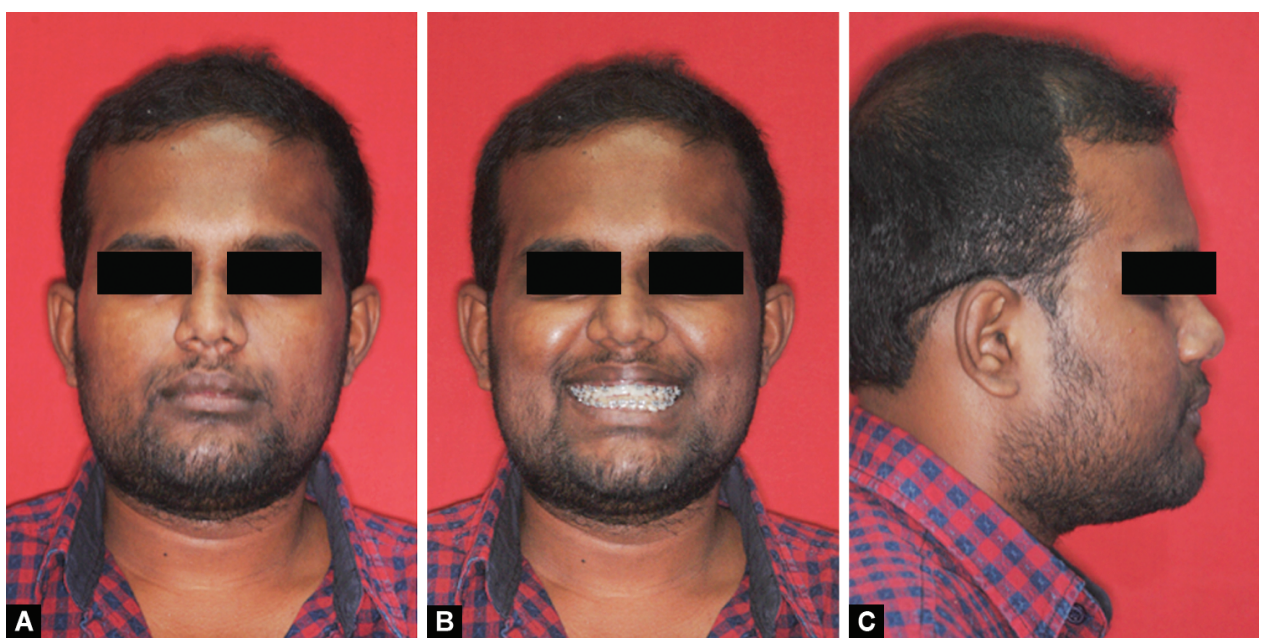

Figs 3 A to C: Immediate postsurgical extraoral photographs 

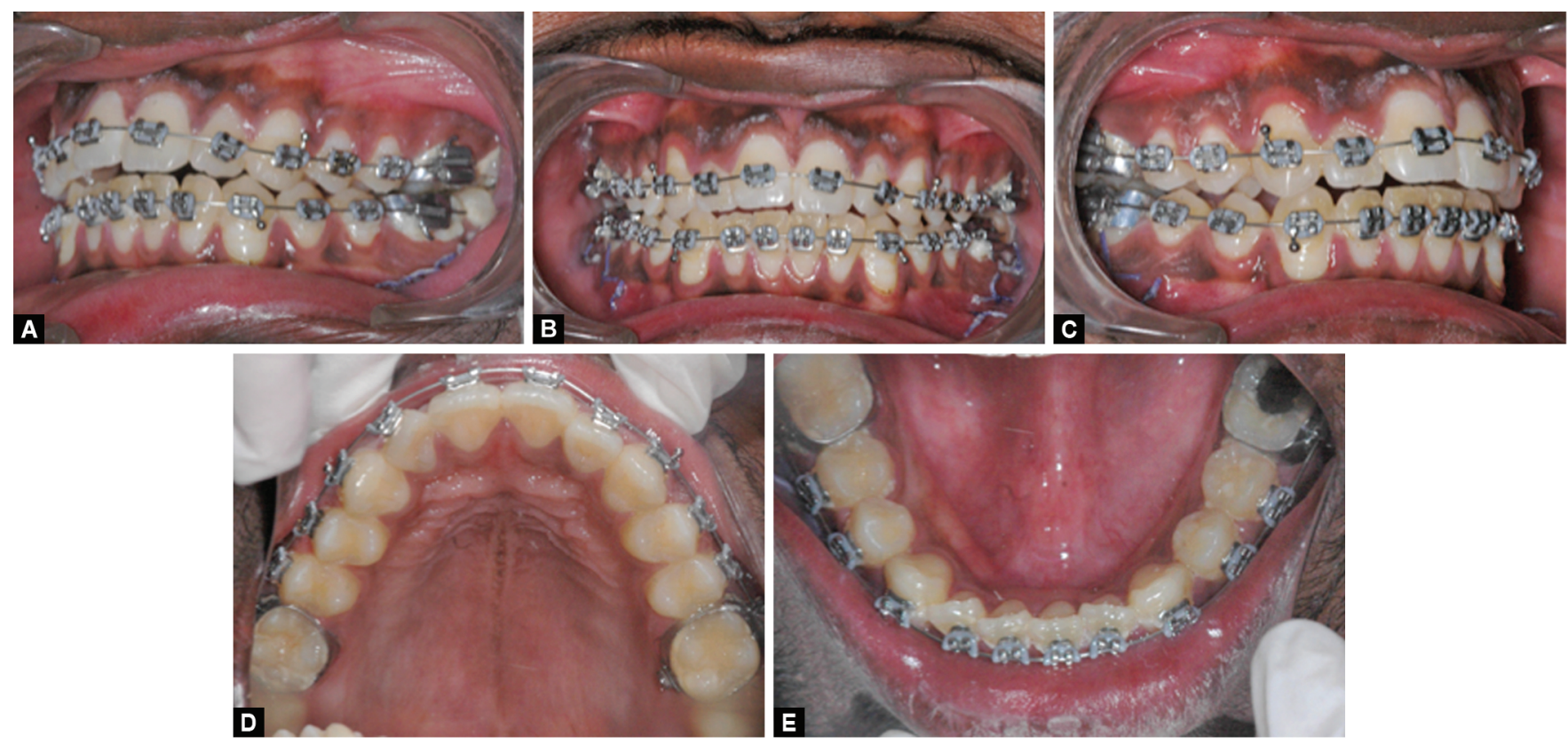

Figs 4A to E: Immediate postsurgical intraoral photographs
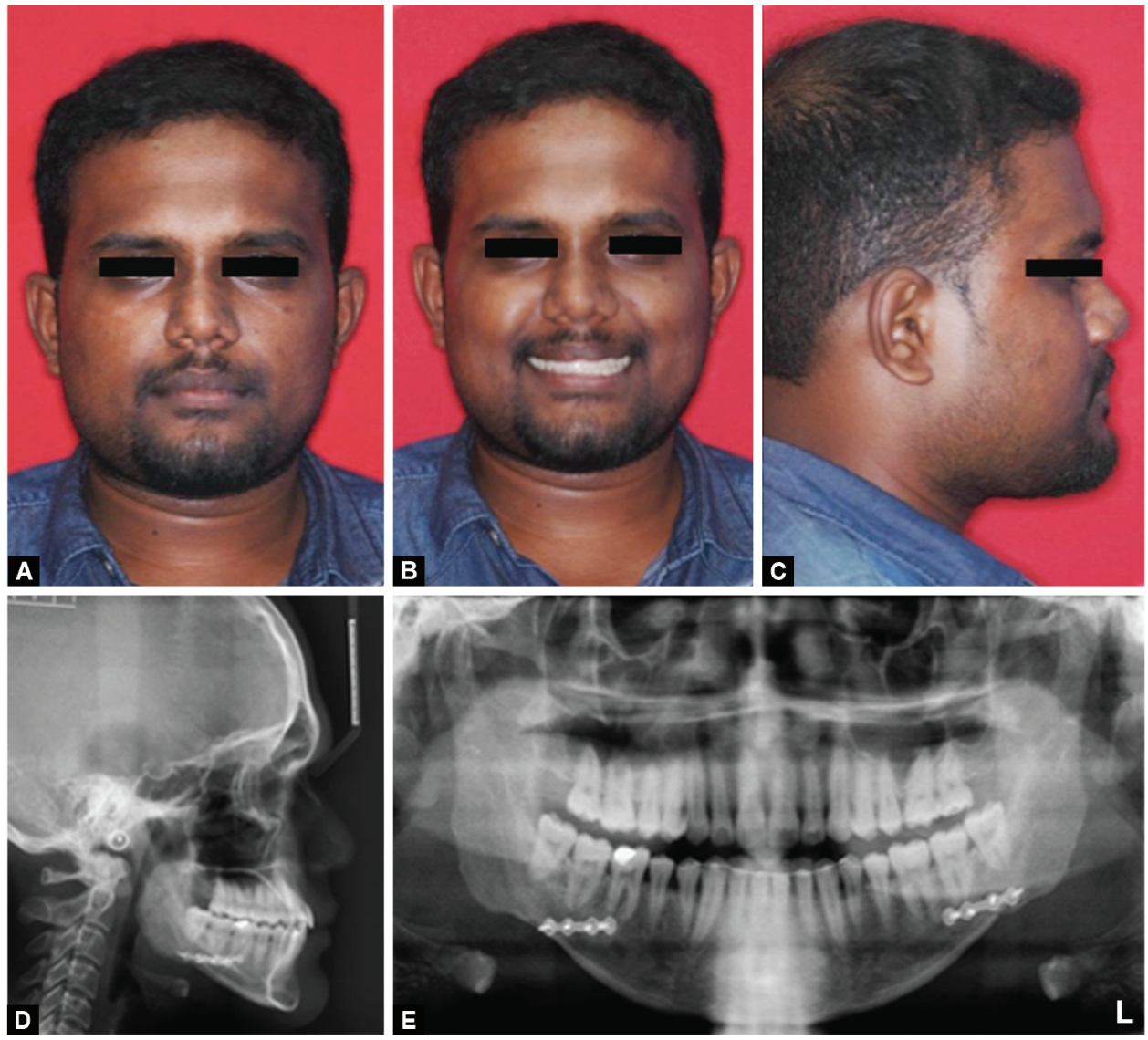

Figs 5A to E: Posttreatment extraoral photographs and radiographs

and existing arch coordination; and (9) the patients should be of appropriate age to proceed with surgery. ${ }^{11}$

The main leverage of any SFA is the high bone metabolic changes that are encountered due to increased osteoclastic activities ultimately resulting in accelerated postoperative tooth movement due to RAP. The RAP is the primary reason for entailing SFOA to significantly shorten the treatment duration with fewer treatment stages when in comparison to conventional orthognathic 

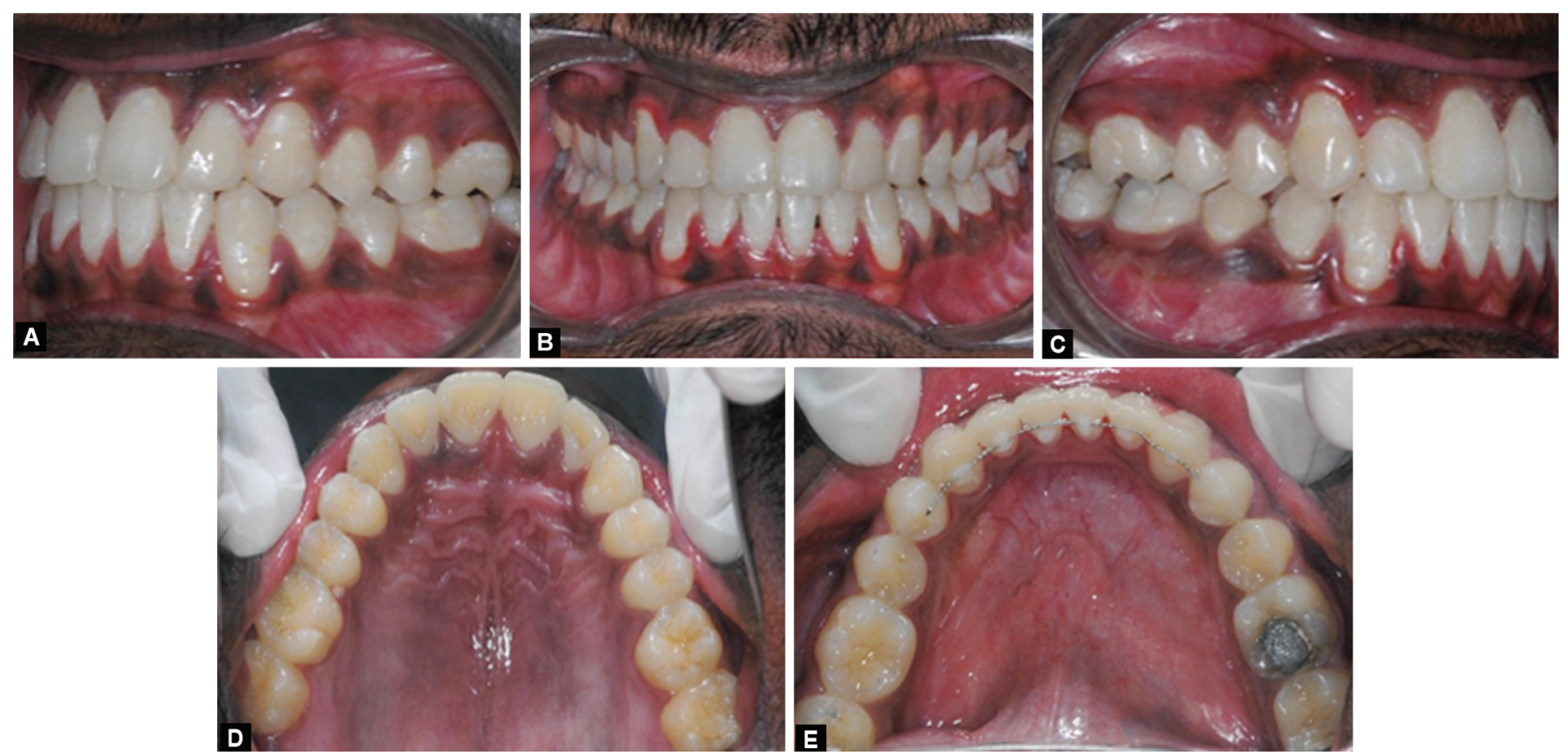

Figs 6 A to E: Posttreatment intraoral photographs

treatment and the possibility of immediate correction of the problem. ${ }^{12}$

Most of the patients with mandibular prognathism associated with open bite are good candidates for the SFA as they usually have mild crowding with less dental compensation. Treating such cases with SFA shows better superior movement than being treated with presurgical orthodontics for point $B$ and Pog as suggested by Liao et al. ${ }^{13}$

Two possible treatment options were described to the patient. The conventional approach that would require presurgical orthodontics followed by surgery and postsurgical orthodontics for finishing but would require a minimum of 20 months' duration. The possibility of SFA with surgery followed by postsurgical orthodontics for finishing and will need a predicted 6-8 months for completion due to utilization of the RAP was given. The patient opted for the SFA as it reduced the treatment duration significantly.

A BSSO with a setback of the mandibular segment by $8 \mathrm{~mm}$ with an anterior pitch up of the mandible by $2 \mathrm{~mm}$ and yaw correction toward the left side by $2 \mathrm{~mm}$ was done to alleviate the open bite, midline shift, and reverse overjet (Figs 3 and 4). Following the surgery, postsurgical orthodontics was carried out to achieve stable occlusion. The treatment duration for the patient only lasted for 5 months and 15 days.

\section{Conclusion}

Posttreatment records showed improved facial esthetics owing to achievement of class I skeletal base, with a symmetrical chin and enhanced lip posture. Class I canine and molar relationships were achieved, along with a levelled maxillary and mandibular occlusal plane with an ideal overjet and overbite (Figs 5 and 6). Superimposition of pretreatment and posttreatment cephalometric tracings confirmed the improvement in mandibular positioning and lower lip posture after the mandibular setback surgery. Post appliance withdrawal, upper and lower Essix was given to the patient in order to maintain the vertical relation of the molars.

\section{Clinical Significance}

This case report demonstrates that the SFA can be successfully used in correcting a severe skeletal class III malocclusion with open bite. Facial appearance and stable occlusion were obtained with reduced treatment duration by eliminating the presurgical phase, utilizing the rapid bone remodeling process, which in turn accelerated tooth movement without noticeable side effects. In addition, worsening of the facial appearance and function customarily noted in the conventional orthognathic approach was sidestepped ensuing in immense patient gratification.

\section{References}

1. Kiyak A, McNeil RW, West RA. The emotional impact of orthognathic surgery and conventional orthodontics. Am J Orthod 1985;88(3): 224-234. DOI: 10.1016/S0002-9416(85)90217-9.

2. Baek SH, Ahn HW, Kwon YH, et al. Surgery-first approach in skeletal class III malocclusion treated with 2-jaw surgery: evaluation of surgical movement and postoperative orthodontic treatment. J Craniofac Surg 2010;21(2):332-338. DOI: 10.1097/SCS.0b013e3181cf5fd4.

3. Nagasaka H, Suguwara J, Kawamura H, et al. "Surgery first" skeletal class III correction using the skeletal anchorage system. J Clin Orthod 2009;43(2):97-105.

4. Villegas C, Uribe F, Sugawara J, et al. Expedited correction of significant dentofacial asymmetry using a "Surgery first" approach. J Clin Orthod 2010;44(2):97-103.

5. Sugawara J, Nagasaka H, Yamada S, et al. The application of orthodontic miniplates to Sendai surgery first. Sem Orthod 2018;24(1):17-36. DOI: 10.1053/j.sodo.2018.01.003.

6. Alfaro FH, Martínez RG, Coral AM, et al. "Surgery first" in bimaxillary orthognathic surgery. J Oral Maxillofac Surg 2011;69(6):201-207. DOI: 10.1016/j.joms.2011.01.010.

7. Brachvogel P, Berten JL, Hausamen JE. Surgery before orthodontic treatment: a concept for timing the combined therapy of skeletal 
dysgnathias. Dtsch Zahn Mund Kieferheilkd Zentralbl 1991;79(7): 557-563.

8. Huang CS, Hsu SS, Chen YR. Systematic review of the surgery-first approach in orthognathic surgery. Biomed J 2014;37(4):184-190. DOI: 10.4103/2319-4170.126863.

9. Nuccio FD, D'Emidio MM, Pelo S. Surgery-first: a new approach to orthognathic surgery. Oral Implantol 2016;9(1):98-102. DOI: 10.11138/ orl/2016.9.1S.098.

10. Behrman SJ, Behrman DA. Oral surgeons' considerations in surgical orthodontic treatment. Dent Clin North Am 1988;32(3):481-507.
11. Sharma VK, Yadav K, Tandon P. An overview of surgery-first approach: recent advances in orthognathic surgery. J Orthod Sci 2015;4(1):9-12. DOI: $10.4103 / 2278-0203.149609$.

12. Choi DS, Garagiola U, Kim SG. Current status of the surgery-first approach (part I): concepts and orthodontic protocols. Maxillofac Plast Reconstr Surg 2019;41(1):10. DOI: 10.1186/s40902-019-0194-4.

13. Liao YF, Lo SH. Surgical occlusion setup in correction of skeletal class III deformity using surgery-first approach: guidelines, characteristics and accuracy. Sci Rep 2018;8(1):11673. DOI: 10.1038/s41598-01830124-2. 